\title{
A Comparison of Central Sensory Reception and Integration Abilities between Older Females with and without Fibromyalgia: A Pilot Study
}

\author{
Yi-Hsin Ko, Debra J. Rose \\ Center for Successful Aging, Department of Kinesiology, California State University, Fullerton, USA \\ Email: drose@fullerton.edu
}

Received 16 February 2015; accepted 6 March 2015; published 12 March 2015

Copyright (C) 2015 by authors and Scientific Research Publishing Inc.

This work is licensed under the Creative Commons Attribution International License (CC BY). http://creativecommons.org/licenses/by/4.0/

(c) (i) Open Access

\begin{abstract}
Purpose: Impaired postural control has been reported in females with fibromyalgia syndrome (FMS). This study aimed to investigate a) differences in central sensory reception and integration abilities for postural control between older females with and without FMS, and b) the influence of a secondary cognitive task on postural control in altered sensory environments. Methods: The performances of 10 older females with FMS were compared to eight age-matched healthy females across single and dual task conditions using the Sensory Organization Test ${ }^{\circledR}$. Results: No significant group differences were evident for Composite Equilibrium Score (CES) in the single task condition ( $p=0.06$ ) but a large effect size (Cohen's $d=0.96$ ) was obtained, suggesting a clinically relevant difference existed between groups with respect to general sensory reception and integration abilities. Single trial Equilibrium Scores (ESs) for both groups declined significantly in the more complex sensory conditions across both task conditions $(p<0.01)$ with a greater decline evident for the FMS group in sensory condition 4 in the dual-task condition $(p=0.04)$. The FMS group also experienced multiple falls in sensory conditions 5 and 6 across both task conditions with a significantly higher percentage of falls evident for the FMS group in condition 6 across both task conditions $(p=\mathbf{0 . 0 4})$. Conclusions: Overall postural stability was significantly lower in the FMS group when compared to an age-matched control group. Postural stability declined further in the FMS group when attempting to perform a secondary cognitive task in both sensory conditions that primarily relied on vestibular inputs for postural control. A greater focus on multisensory and dual-task training in a balance environment may benefit older females with FMS.
\end{abstract}

\section{Keywords}

Fibromyalgia Syndrome, Older Adults, Sensory Organization Test ${ }^{\circledR}$, Dual-Task 


\section{Introduction}

Fibromyalgia syndrome (FMS) is a chronic pain disorder that is characterized by widespread musculoskeletal pain, stiffness, fatigue, in addition to other bodily symptoms [1]-[4]. FMS can be triggered by genetic factors, environmental stressors, or neurophysiological changes [5] [6]. The prevalence of FMS among adults 60 years and older has been estimated at 5\% to 8\% in females; a higher percentage than observed in younger age groups and male adults [7] [8]. Several studies have shown its adverse effect on both static and dynamic postural control in adults [2] [4] [9]-[11]. In a recent survey of 2596 adults ranging in age from 17 to 78 years, approximately $45 \%$ of adults with FMS self-reported balance problems, which was one of the most commonly reported symptoms [2].

Previous research has demonstrated that adults with FMS have significant central sensory impairment when compared to age-matched healthy adults [9]-[11] whereas only small and non-significant differences were evident for motor function [10]. It has been argued that the chronic musculoskeletal pain associated with FMS may result in altered sensory function [12]-[14]. Bayazit et al. [12] have also suggested that neural brainstem disintegration may explain some of the significant changes in sensory function in persons with FMS. These changes may, in turn, adversely affect postural control, particularly in individuals with FMS. In the present study, the Sensory Organization Test ${ }^{\circledR}$ (SOT) [15] was used to examine central sensory reception and integration abilities in older adults, with and without FMS. The SOT provides a comprehensive evaluation of central sensory reception and integration abilities and can identify specific impairments in each of the three sensory systems (i.e., vision, somatosensory, vestibular) that contribute to balance. This test has been widely used to evaluate central sensory function across a number of different populations, including older adults [16]-[18].

Higher order cognitive abilities such as attention, working memory, and information-processing speed are essential for maintaining postural stability [19]. Limited attentional resources may increase the risk of impaired balance function in older adults [20]. With normal aging, both the quantity and quality of sensory information derived from the visual, somatosensory, and/or vestibular systems that sub serve postural control declines. As a result, higher levels of attentional focus must be directed to maintaining postural stability [20] [21]. The attentional demands become even greater when the availability of visual, somatosensory, and/or vestibular inputs are reduced [21] [22]. Postural sway has also been shown to increase significantly in healthy older adults when the available sensory information is reduced [22] or they are required to perform a secondary task while maintaining balance [20]. Several studies have demonstrated that adults with FMS experience significant changes in attention and working memory [23]-[25]. These changes in higher order cognitive processes in older adults with FMS may further limit the available attentional resources for postural control in dual-task environments, particularly when the availability of sensory information is also altered.

Although several studies have shown impaired postural control in adults with FMS, none, to our knowledge, have investigated central sensory reception and integration abilities in older adults with FMS when compared to age-and gender-matched healthy older adults. Moreover, no studies have investigated how the performance of a secondary cognitive task in altered sensory environments further influences postural control in older adults with FMS. Our primary goal in this study, therefore, was to investigate whether differences in central sensory reception and integration abilities were evident between older women, with and without fibromyalgia. A secondary goal was to further examine the influence of an additional cognitive task on postural control across different sensory environments between women, with and without FMS.

\section{Methods}

\subsection{Participants}

Females aged 60 years and older with a diagnosis of FMS were recruited to participate in this case-control study. Eligible participants were contacted through a local fibromyalgia research center. A diagnosis of fibromyalgia was confirmed via phone using the screening criteria established by the American College of Rheumatology (ACR) in 2010 [1]. Eligible participants had to reside in the community, be functionally independent, speak English as their primary language, and had completed a minimum of high school or higher level of education. Participants were excluded if they were currently experiencing musculoskeletal and/or neurological diseases that might interfere with their performance on the balance test. Age- and gender-matched healthy control (HC) participants were recruited for comparison purposes. A Health/Activity Information Questionnaire (HAQ) was ad- 
ministered during the phone call to ensure that all participants met the eligibility criteria and had no other medical condition(s) (e.g., neurological or significant musculoskeletal conditions, major depression, diabetes, or uncorrected visual problems.) that might adversely affect balance. Participants reporting any contraindicated medical conditions were excluded from participation in the study. While no formal screening for cognitive impairment was conducted, all participants were required to report any diagnosis of cognitive impairment on the study-related HAQ, complete all associated paperwork without assistance, and demonstrate their ability to perform two trials of the cognitive task alone prior to the start of the dual-task condition. This study was approved by the California State University Fullerton, Institutional Review Board (FWA00015384), and all participants reviewed and signed the approved informed consent form prior to testing.

\subsection{Questionnaires/Surveys}

Demographic information and a comprehensive medical history were obtained from each participant using a HAQ specifically designed for the present study. History of the diagnosis and symptoms of FMS was also obtained for the FMS group. The Revised Fibromyalgia Impact Questionnaire (FIQR) and the Symptom Impact Questionnaire (SIQR) [3] were used to measure FMS-related impact in the FMS and more general symptom impact in the HC groups, respectively. Participants rated their perceived level of function and symptom severity using a zero to 10 scoring system. All questions were answered based on the average condition experienced during the past 7 days. A higher score indicated a higher level of functional limitation or a higher level of symptom intensity.

\subsection{Apparatus}

In order to evaluate central sensory reception and integration abilities, both groups completed the Sensory Organization Test ${ }^{\circledR}$ (SOT) on the SMART Balance Master system (NeuroCom International Inc., Clackamas, Oregon), version 8.2. The SOT is considered to be the "gold standard" for assessing central sensory reception and integration abilities. By sway-referencing the support surface and/or visual surround during the administration of the $\mathrm{SOT}^{\circledR}$, an individual's ability to effectively use sensory inputs while suppressing inaccurate sensory information from the visual, somatosensory, and/or vestibular systems can be quantified. Six specific sensory conditions were presented during the SOT: standing with eyes open on a stable surface; standing with eyes closed on a stable surface; standing with eyes open with sway-referenced vision on a stable surface; eyes open while standing on a sway-referenced surface; eyes closed while standing on a sway-referenced surface; and eyes open with sway-referenced vision while standing on a sway-referenced surface [15] (Figure 1). Participants were

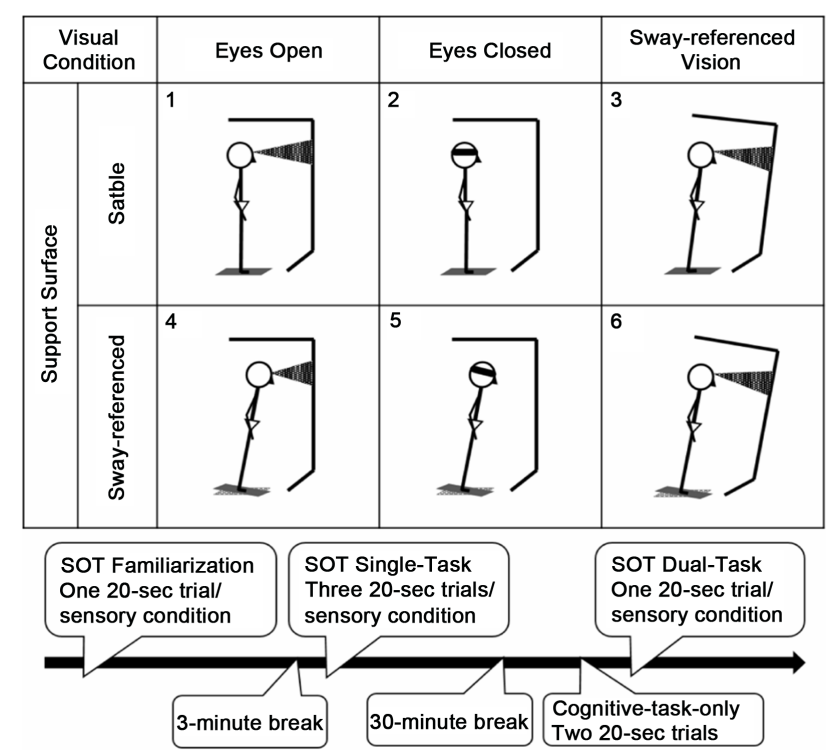

Figure 1. The six sensory conditions of the sensory organization test ${ }^{\circledR}$ (Adapted from Neuro Com International Inc., Clackamas, Oregon) and testing procedure. 
instructed to stand quietly during each test trial. An Equilibrium Score (ES) was recorded for each of three 20-second trials. The ES was calculated based on the degree of excursion of the individual's center of pressure. The score ranged from zero to 100 points; a higher score indicated better postural stability. A weighted average score, the Composite Equilibrium Score (CES), was then calculated across the six sensory conditions [26]. Finally, falls were recorded for any test trials in which a participant lost balance and required assistance from the overhead harness and/or test administrator. The test-retest reliability of the SOT has previously been established in a sample of non-institutionalized older adults [18] as well as adults with fibromyalgia [27].

\section{Secondary Cognitive Task}

In order to examine how cognitive load influences central sensory reception and integration abilities in older adults, with and without FMS, a word-generation task served as the secondary task [28] [29]. In the dual-task condition, participants were asked to call out as many words starting with a designated letter as they could during each standing trial. For the dual-task condition, participants performed a single 20 -second trial in each of the six sensory conditions. All participants were presented with the same sequence of six different letters (B, S, D, C, $\mathrm{A}, \mathrm{W}$ ) for the six dual-task balance trials. Two cognitive-task only trials were practiced prior to having the participants perform the task during performance of the SOT ${ }^{\circledR}$. The number of correctly spoken words was recorded using an audio recorder to ensure accuracy of spoken words during data collection.

\subsection{Procedures}

On the scheduled testing day, participants completed the FIQR or the SIQR. Based on the outcomes of pilot testing conducted with two older females with FMS, both the single-task and dual-task SOTs were conducted on a single day of testing. Prior to administering the first SOT, a familiarization test was conducted. This familiarization test was intended to assist the participant become more familiar with the equipment, testing protocol, and overhead safety harness. This session was also helpful for obtaining reliable data and reducing the participant's anxiety level [27]. A three-minute rest interval was provided between the familiarization test and the single-task condition of the SOT. During the single-task SOT, three 20-second trials in each of the six sensory conditions were completed. Participants then completed the dual-task condition of the SOT after a 30-minute rest interval. During performance of the second SOT, participants were required to perform the word generation task while standing in each of the six sensory conditions. In order to minimize learning effects, only one trial was performed in each of the six sensory conditions in the dual-task condition (Figure 1).

A fall was recorded if the participant needed external support to avoid losing balance, moved her feet, or prematurely opened her eyes during the eyes-closed conditions. The trial was stopped immediately if any of these circumstances occurred and a fall was recorded. A report card was provided and the results were explained to each participant after the completion of the two SOTs. The duration of the single testing session was approximately 90 minutes.

\subsection{Data Analyses}

Descriptive analyses were performed for the demographics, medical conditions, and symptom data. Betweengroup differences for the variables of age, number of medical conditions, and FIQR/SIQR scores were analyzed using independent t-tests while between-group comparisons for the variables of presence of leg symptoms (i.e. swelling, numbness, tingling), fear-of-falling, and fall history were analyzed using Chi-Squared tests.

In order to explore differences in central sensory reception and integration abilities, the CES for the single-task condition was compared between groups using an independent t-test analysis. Additionally, a 2 (group) $\times 6$ (sensory condition) ANOVA for the mean ES in the single-task condition was also conducted to determine whether group differences were evident across the six sensory conditions. A 2 (group) $\times 2$ (task condition) $\times 6$ (sensory condition) repeated measures analysis of variance (RM ANOVA) was then conducted to investigate within- and/or between-group differences between the single- and dual-task condition and across the six sensory conditions. Only the ES for the first trial in each of the sensory conditions was included in this analysis. The number of correctly spoken words during performance of the word generation task was analyzed using a 2 (group) $\times 6$ (sensory condition) ANOVA. In the case of significant findings, post-hoc analyses using t-tests with Bonferroni adjustments were conducted to explore the direction of the significant findings. In order to ascertain the practical significance of certain group comparisons, effect sizes were also calculated. Finally, the number of 
falls recorded during the first SOT trial for each group was also analyzed across the two task conditions. Withingroup comparisons were analyzed using Friedman's ANOVA. In the case of significant findings, post-hoc Wilcoxon Signed-Rank Tests were conducted. Between-group comparisons were analyzed using Chi-Squared Tests.

All statistical analyses were conducted using the Statistical Package for the Social Sciences (SPSS) software, version 19.0. A statistical significance level of $p<0.05$ was used for all analyses conducted.

\section{Results}

Ten older females with FMS and eight age-matched healthy females were included in the analyses. No significant group differences were observed for the variables of age (mean age \pm SD for the FMS group: $65.49 \pm 4.72$ years; HC group: $66.08 \pm 4.83$ years), leg symptoms experienced, fear of falling, or fall history. As expected, the number of medical conditions for the FMS group was significantly higher than the HC group $(p=0.03)$. Higher weighted total scores were also evident for the FMS group (mean \pm SD: $30.12 \pm 24.31$, score range 6 to 75 ) on the FIQR/SIQR when compared to the HC group (mean \pm SD: $7.46 \pm 11.64$, score range 0 to $35, p<0.05$ ). Specifically, the mean subtotal score for each of the three domains was significantly higher for the FMS group when compared to the HC group, indicating a significantly higher impact of symptoms on the performance of daily living activities $(p<0.01)$, significantly lower perceived ability to accomplish goals of the week $(p=0.04)$, and significantly higher perceptions of symptom intensity $(p<0.01)$ in the FMS group (Table 1$)$.

The results of an independent t-test failed to demonstrate a significant statistical difference in CES between the FMS and HC groups (Mean \pm SD: $67.70 \pm 13.09$ and $77.25 \pm 5.09$, respectively; $p=0.06$ ) in the single task condition. The calculated effect size, however, was large (Cohen's $d=0.96$ ). Due to the high number of falls recorded by the FMS group on one or more trials in sensory conditions 5 and 6 in the single task condition (Table 2), only the mean ESs for the first four sensory conditions were analyzed using a 2 (Group) $\times 4$ (Sensory Condition) RM ANOVA. The only significant finding emerging from this analysis was a main effect for Sensory Condition $\left(p<0.01, \eta^{2}=0.59\right.$ ). Post-hoc analyses conducted to further explore the source(s) of the main effect for Sensory Condition revealed significant differences $(p<0.01)$ for all pairwise comparisons with the exception of pairwise comparisons between sensory condition 2 (eyes closed, firm surface) and 3 (sway-referenced surface, eyes open) (Figure 2).

Table 1. Demographics and medical history.

\begin{tabular}{|c|c|c|c|}
\hline & FMS Group $(\mathrm{N}=10)$ & HC Group $(\mathrm{N}=8)$ & $p$ value \\
\hline Age (Mean (SD)) & $65.49(4.72)$ & $66.08(4.83)$ & 0.80 \\
\hline Number of Medical Conditions (Mean (SD)) & $4.90(2.69)$ & $2.38(1.69)$ & 0.03 \\
\hline \multicolumn{4}{|l|}{ Years of FMS Diagnosis (Number) } \\
\hline $6-10$ Years & 3 & 0 & \\
\hline 11 - 15 Years & 3 & 0 & \\
\hline 16 - 20 Years & 3 & 0 & \\
\hline Greater than 20 Years & 1 & 0 & \\
\hline Leg Symptoms (Numbness, Tingling, Swelling) (Number) & 7 & 3 & 0.17 \\
\hline Fear of Falling (Moderate to Extreme) (Number) & 4 & 2 & 0.50 \\
\hline Fall History in Previous 12 Months (Number) & 5 & 1 & 0.09 \\
\hline \multicolumn{4}{|l|}{ FIQR/SIQR (Mean (SD)) } \\
\hline Weighted Total Score & $30.12(24.31)$ & $7.46(11.64)$ & 0.02 \\
\hline Domain 1: Limitation in Daily Physical Activity & $28.70(22.72)$ & $2.88(6.17)$ & $<0.01$ \\
\hline Domain 2: Overall Impact of Symptoms & $5.90(6.03)$ & $0.75(2.12)$ & 0.04 \\
\hline Domain 3: Symptom Intensity & $49.10(20.84)$ & $11.50(15.50)$ & $<0.01$ \\
\hline
\end{tabular}

Note: FMS, participants with fibromyalgia syndrome; HC, healthy controls; FIQR, revised fibromyalgia impact questionnaire; SIQR, revised symptom impact questionnaire. 


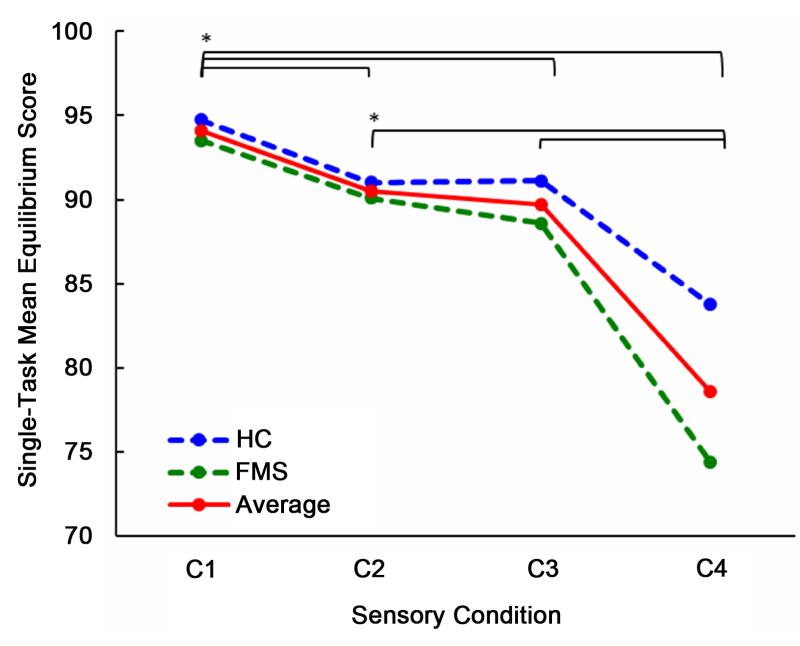

Figure 2. Mean equilibrium scores as a function of group and sensory condition.

Table 2. Fall occurrences in the FMS group.

\begin{tabular}{|c|c|c|c|c|c|c|c|c|c|}
\hline \multirow{2}{*}{ Sensory Condition } & \multicolumn{3}{|c|}{ Condition 4} & \multicolumn{3}{|c|}{ Condition 5} & \multicolumn{3}{|c|}{ Condition 6} \\
\hline & Trial 1 & Trial 2 & Trial 3 & Trial 1 & Trial 2 & Trial 3 & Trial 1 & Trial 2 & Trial 3 \\
\hline \multicolumn{10}{|l|}{ Single Task } \\
\hline Participant ID & None & $\begin{array}{c}\text { FM5 } \\
\text { FM12 }\end{array}$ & FM5 & $\begin{array}{l}\text { FM5 } \\
\text { FM4 } \\
\text { FM9 }\end{array}$ & $\begin{array}{c}\text { FM5 } \\
\text { FM12 }\end{array}$ & FM5 & $\begin{array}{l}\text { FM5 } \\
\text { FM4 } \\
\text { FM9 } \\
\text { FM6 }\end{array}$ & $\begin{array}{c}\text { FM5 } \\
\text { FM13 }\end{array}$ & $\begin{array}{l}\text { FM5 } \\
\text { FM4 } \\
\text { FM9 }\end{array}$ \\
\hline \multicolumn{10}{|l|}{ Dual Task } \\
\hline Participant ID & None & - & - & $\begin{array}{c}\text { FM5 } \\
\text { FM4 } \\
\text { FM12 }\end{array}$ & - & - & $\begin{array}{c}\text { FM5 } \\
\text { FM4 } \\
\text { FM12 } \\
\text { FM6 }\end{array}$ & - & - \\
\hline
\end{tabular}

As was the case for the single task condition, four participants in the FMS group experienced a fall on the first and only trial of sensory conditions 5 and/or 6 in the dual-task condition (Table 2). Therefore, a 2 (Group) $\times 2$ $($ Task) $\times 4$ (Sensory Condition) RM ANOVA was conducted. A three-way interaction between Task, Sensory Condition, and Group $\left(p=0.04, \eta^{2}=0.18\right)$ and a two-way interaction between Task and Group $\left(p=0.03, \eta^{2}=\right.$ $0.26)$ were evident. Significant main effects for Task $\left(p=0.06, \eta^{2}=0.20\right)$ and Sensory Condition $\left(p<0.01, \eta^{2}=\right.$ 0.74) were also evident (Table 3). The three-way interaction is visually represented in Figure 3(a) and Figure 3(b). Subsequent post-hoc analyses to identify the source(s) of the interaction effect revealed that both groups demonstrated a lower ES in sensory condition 1 in the dual- versus single-task condition $(p<0.01)$ whereas ES was significantly lower for the FMS group during condition 4 in the dual-task condition $(p<0.01)$. No significant group or task condition differences were evident for the remaining two sensory conditions. No significant within- or between-group differences were found for the number of correctly spoken words across the six sensory conditions presented in the dual-task condition.

The results of a chi-squared analysis conducted to investigate group differences with respect to falls indicated that the FMS group recorded a significantly higher percentage of falls in sensory condition 6 in both the single and dual task conditions when compared to the HC group $(p<0.05)$. A closer review of the between-group data further revealed that six participants in the FMS group experienced falls on one or more trials in sensory conditions 4, 5, and/or 6 in the single task condition whereas no participants in the HC group experienced any falls in either the single or dual-task conditions. In the dual-task condition, three participants in the FMS group fell on the first trial of sensory condition 5 , and four participants fell on sensory condition 6 . The results of a 1 (FMS Group) $\times 6$ (Sensory Condition) Friedman’s ANOVA revealed significant differences in the percentage of falls 


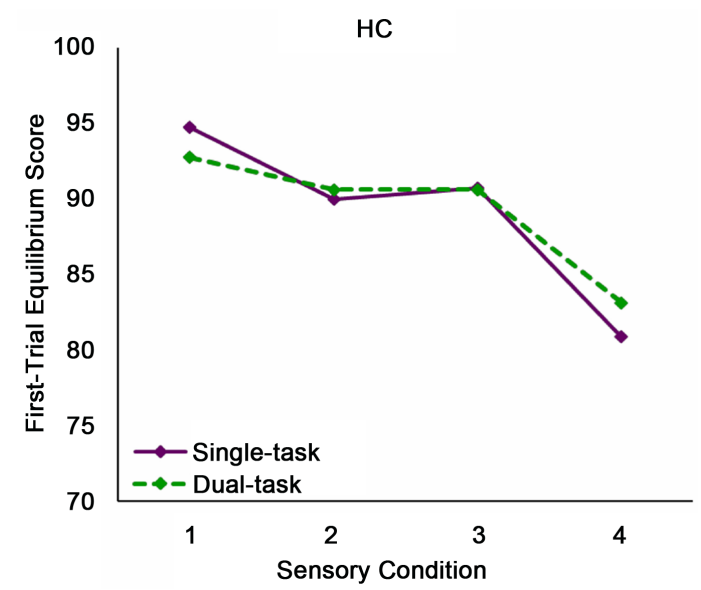

(a)

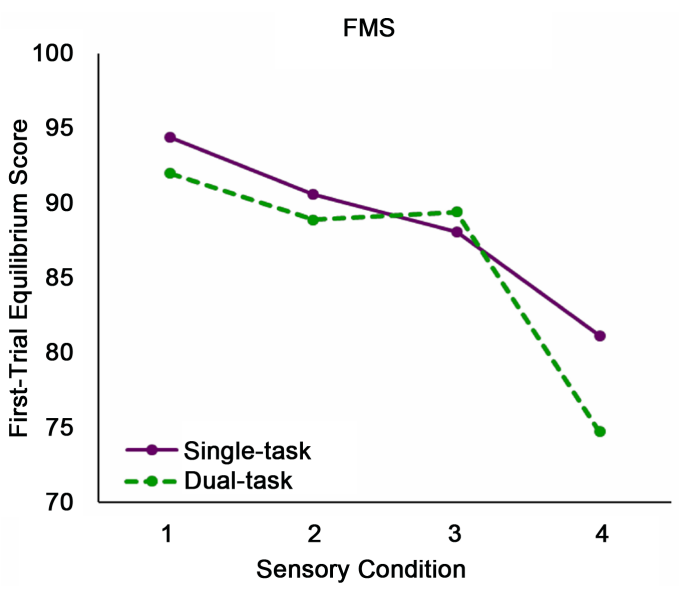

(b)

Figure 3. Interaction between task condition, sensory condition, and group. (a) Performance of participants with FMS; (b) Performance of participants without FMS.

Table 3. Results of the 2 (group) $\times 2$ (task condition) $\times 4$ (sensory condition) Factorial ANOVA for single trial equilibrium score across task conditions.

\begin{tabular}{ccccc}
\hline Comparisons & $F$ & $p$ & Partial $\eta^{2}$ & Power \\
\hline Task Condition $\times$ Sensory Condition $\times$ Group & 3.50 & 0.04 & $0.18^{*}$ & 0.62 \\
Task Condition $\times$ Group & 5.57 & 0.03 & $0.26^{*}$ & 0.60 \\
Sensory Condition $\times$ Group & 0.95 & 0.37 & 0.06 & 0.17 \\
Task Condition $\times$ Sensory Condition & 1.29 & 0.29 & 0.08 & 0.26 \\
Task Condition & 4.01 & 0.06 & $0.20^{*}$ & 0.47 \\
Sensory Condition & 45.84 & $<0.01$ & $0.74^{*}$ & 1.00 \\
Group & 2.35 & 0.15 & 0.13 & 0.30 \\
\hline
\end{tabular}

Note: ${ }^{*}$ indicates large effect $\left(\eta^{2}>0.14\right)$.

recorded within the FMS group $(p<0.01)$. Subsequent post-hoc analyses using Wilcoxon signed-rank tests further indicated that the percentage of falls in sensory condition 6 in both the single and dual task conditions was significantly higher for the FMS group than the percentage of falls in any other sensory condition with the exception of sensory condition $5(p<0.05)$.

\section{Discussion}

The results from this study are consistent with previous studies [9]-[11], but also yielded some new findings. When compared to the age-matched HC group, the FMS group experienced 1) a higher perceived level of FMSrelated symptoms and functional limitations; 2) lower overall postural stability in the single task condition; 3) significantly lower postural stability in sensory condition 4 in the dual-task condition; and 4) a significantly higher number of falls in sensory conditions 5 and 6 across both task conditions.

To our knowledge, very few studies have measured central sensory reception and integration abilities in older adults with FMS using the SOT $^{\circledR}$. Jones et al. [10] used the SOT to assess postural control ability in middle-aged adults (48.6 \pm 9.7 years), with and without FMS, while Russek and Fulk [11] conducted the SOT in a sample of females (20 to 89 years) with FMS and compared their overall performance to normative data. The findings of both studies identified impaired central sensory reception and integration abilities in females with FMS on the basis of a significantly lower CES. Although group differences in CES recorded for the single task condition just failed to reach statistical significance $(p=0.06)$ in the present study, a large effect size was obtained (Cohen's $d$ $=0.96$ ) when CES was compared between the two groups. Unlike statistical power, which is heavily influenced 
by sample size, effect size calculations are not. The practical or clinical significance of our study findings could therefore be explored using this method of analysis in spite of low statistical power (0.48).

In contrast to the group differences observed for CES in the single task condition, no group differences in mean ES (single task condition) were evident across the first four sensory conditions in the present study. Instead, both groups demonstrated significantly higher levels of postural instability when either visual (sensory conditions 2 and 3) or somatosensory (sensory condition 4) inputs were manipulated when compared to sensory condition 1. Unlike the results obtained by Jones et al. (2011) who found significant group differences across the first four sensory conditions, both groups in the present study demonstrated higher levels of postural instability as the sensory conditions became more complex. This difference is likely due to the fact that both groups in our study were much older ( $M=66$ years) than the participants in the earlier study ( $M=49$ years). Nevertheless our findings for the FMS group are consistent with those reported by Jones et al.

Analyzing fall incidence rates across the six sensory conditions in the single task condition further identified group differences in postural stability. The FMS group experienced a higher number of falls in conditions $5(\mathrm{~N}=$ $3)$ and $6(\mathrm{~N}=4)$ when compared to the HC group with the difference being statistically significant in sensory condition 6 only. In contrast, the HC group experienced no falls in any of the six sensory conditions. These results suggest that older adults with FMS are unable to appropriately weight sensory system input when the vestibular system is the primary source of sensory information for upright balance. This deficit may place them at a higher risk for falls, particularly on initial exposure to sensory conditions in which vision and somatosensory inputs are no longer providing accurate input (e.g., low light conditions combined with unstable surfaces). Our findings partially support those of Russek and Fulk [11] and Jones et al. [10] who identified significant impairments in both the visual and vestibular systems in their participants with FMS. These authors suggested that the differences may be the result of either an abnormal use of postural strategies (i.e., ankle versus hip in the more complex sensory conditions) or neuromuscular incoordination due to elevated pain levels in the lower legs. It is interesting to note that the women with FMS who fell in conditions 5 and 6 in the present study recorded higher, albeit non-significant, FIQR total scores when compared to women with FMS who did not fall in either sensory condition. Jones et al. also found that women with FMS who reported a higher level of FMS-related symptoms demonstrated lower levels of overall postural stability. How the level of symptom severity may affect their ability to appropriately weight incoming sensory information in changing sensory environments warrants further investigation.

Attentional demands for postural control increase when the sensory environment becomes more complex, particularly in older adults [21] [22]. While a person's attentional capacity is limited, the ability to reallocate attention from a secondary cognitive task to the primary balance task is important for maintaining postural stability in complex sensory environments. The results of this study suggest that greater postural instability is evident for older adult females with FMS when compared to age-matched healthy females, particularly when performing a cognitive task in complex sensory environments. Although no group differences were evident with respect to postural sway across the first three sensory conditions in the dual-task condition, the FMS group demonstrated significantly higher sway when compared to the HC group on sensory condition 4, a condition in which visual inputs become the primary source of information for standing balance. The FMS group also recorded a higher incidence of falls in sensory conditions 5 and 6 when compared to the HC group. These results are similar to those found for the single task condition.

Shumway-Cook et al. [20] advanced the idea of a "posture first" attentional hierarchy to account for the differences observed in their study comparing postural sway between groups of older adults, with and without a history of falls, across different sensory (i.e. firm vs. compliant surfaces) and dual-task conditions (i.e., line match vs. sentence completion tasks). According to the authors, maintaining balance should be the first priority for attentional resources when a person faces a situation where the threat of losing balance is high. While there was no significant between-group differences found for performance on either cognitive task in their study, the group with a history of falls demonstrated significantly higher postural sway when compared to the group with no fall history, particularly during the dual-task condition. That is, older adults with a history of falls failed to adopt a "posture first" strategy when performing a secondary task in an unstable environment.

Similar to the findings of Shumway-Cook et al., no group differences were evident for the number of words spoken across the two task conditions in the present study but higher levels of postural sway and/falls were evident for the FMS group, particularly in sensory conditions 4, 5, and 6. Participants with FMS who experienced higher instability or actually lost their balance during conditions 5 and 6 , were still attempting to generate words 
in the dual-task condition, suggesting an inability to switch their attention from the cognitive to balance task to avoid becoming unstable. At a practical level, the inability to adopt a "posture first" strategy when balance is threatened will likely result in greater risk for falling.

The word generation task used in the present study clearly created a distinct attentional load for both groups as indicated by the fact that both groups recorded significantly higher levels of postural instability in condition 1 (eyes open, firm surface) when the secondary cognitive task was introduced. In addition, the FMS group experienced even greater postural instability than the HC group in sensory conditions 4, 5, and 6 in the dual-task condition. While no group differences were evident for condition 4 in the single-task condition, the FMS group experienced greater postural instability in this condition in the dual-task condition. In this sensory environment, input from the somatosensory system is no longer accurate, making the visual system the primary source of sensory information for upright balance. Jones et al. [10] and Russek and Fulk [11] also suggested that people with FMS may have some level of sensory perception and integration impairments in the visual system based on the findings of their studies. Further study is needed to investigate how different cognitive impairments affect central sensory reception and integration abilities in older adults with FMS.

The findings of earlier studies [10] [11], as well as those emerging from the present study, provide further evidence for impaired central sensory reception and integration abilities in the FMS population. Being able to accurately process and weight incoming sensory information is critical to perceiving where the body is in space and/or responding appropriately in a changing sensory environment. Real-world examples include walking across compliant surfaces in conditions of low lighting, moving through crowds or along busy sidewalks, and/or transitioning between moving and stable surfaces. Although different types of exercise interventions (e.g., tai chi, balance and strength) lead to improvements in balance and reduced fall risk, few systematically target sensory reception and integration [30]. Older adults with FMS may clearly benefit from rehabilitation programs that systematically target the sensory systems, first in single task conditions and then dual-task situations that simulate the types of activities and environments experienced on a daily basis [19]. These recommendations are similar to those proposed by Jones et al. [10] who also suggested that clinicians and exercise instructors include sensory orientation training in their exercise programs, particularly those aimed at lowering fall incidence rates among older adults.

Certain limitations must be noted for the present study. First, the sample of older women who met the eligibility criteria for the study or were willing to participate was relatively small. Difficulties recruiting communityresiding older adults with FMS for the study, coupled with the need to eliminate three participants, one from the FMS group and two from the HC group due to extreme scores or missing data, clearly lowered the statistical power needed to identify group differences. Despite this limitation, however, significant differences were evident when central sensory reception and integration abilities were evaluated in the two groups. Second, only one trial was performed in each of the six sensory conditions in the dual-task condition. While our decision to evaluate performance on a single trial was to minimize learning effects related to the cognitive task, it did not enable us to see whether participants adapted their balance performance in the dual-task situation as the task was repeatedly performed. Data from a single trial in each sensory condition does mimic real life situations wherein the individual must correctly weight incoming sensory inputs from the vestibular, visual, and somatosensory systems on first exposure to a new sensory environment in order to maintain balance. Our results suggest that for older women with FMS this is a particularly difficult task to accomplish, particularly when the vestibular system becomes the primary source of sensory information for controlling upright balance. When the attentional demands are increased by adding a cognitive load, older women with FMS also experience difficulty accurately weighting visual and vestibular inputs to control upright balance.

\section{Conclusion}

The present study demonstrated that overall levels of postural stability were lower in a group of older women with FMS when compared to an age-matched control group as indicated by the large effect size for CES. Regardless of whether the task was to maintain a standing balance position in either a single or dual-task condition, the FMS group recorded a higher percentage of falls on the first trial in each of the two sensory conditions that required the use of the vestibular system to control standing balance. This finding suggests that women with FMS are particularly vulnerable to a loss of balance on first exposure to this type of sensory environment. Moreover, in the dual-task condition, older women with FMS demonstrated significantly higher postural insta- 
bility when performing a cognitive task in sensory conditions that required vision or the vestibular system to serve as the primary source of sensory information for maintaining balance. Balance training programs aimed at reducing the level of fall risk in older women with FMS should include a strong focus on improving central sensory reception and integration skills by constructing practice environments that require both the reweighting of sensory inputs as the different sensory systems are manipulated. Progressively increasing the cognitive load during the concurrent performance of different balance activities may also facilitate the development of improved attentional-switching skills.

\section{References}

[1] Wolfe, F., Clauw, D.J., Fitzcharles, M.A., Goldenberg, D.L., Katz, R.S., Mease, P. and Yunus, M.B. (2010) The American College of Rheumatology Preliminary Diagnostic Criteria for Fibromyalgia and Measurement of Symptom Severity. Arthritis Care and Research, 62, 600-610. http://dx.doi.org/10.1002/acr.20140

[2] Bennett, R.M., Jones, J., Turk, D.C., Russell, I.J. and Matallana, L. (2007) An Internet Survey of 2596 People with Fibromyalgia. BMC Musculoskeletal Disorders, 8, 27. http://dx.doi.org/10.1186/1471-2474-8-27

[3] Bennett, R.M., Friend, R., Jones, K.D., Ward, R., Han, B.K. and Ross, R.L. (2009) The Revised Fibromyalgia Impact Questionnaire (FIQR): Validation and Psychometric Properties. Arthritis Research and Therapy, 11, R120. http://dx.doi.org/10.1186/ar2783

[4] Watson, N.F., Buchwald, D., Goldberg, J., Noonan, C. and Ellenbogen, R.G. (2009) Neurologic Signs and Symptoms in Fibromyalgia. Arthritis \& Rheumatology, 60, 2839-2844. http://dx.doi.org/10.1002/art.24772

[5] Clauw, D.J. (2009) Fibromyalgia: An Overview. American Journal of Medicine, 122, S3-S13. http://dx.doi.org/10.1016/j.amjmed.2009.09.006

[6] Fitzcharles, M.A. and Yunus, M.B. (2012) The Clinical Concept of Fibromyalgia as a Changing Paradigm in the Past 20 Years. Pain Research and Treatment, 2012, Article ID: 184835. http://dx.doi.org/10.1155/2012/184835

[7] White, K.P., Speechley, M., Harth, M. and Ostbye, T. (1999) The London Fibromyalgia Epidemiology Study: The Prevalence of Fibromyalgia Syndrome in London, Ontario. The Journal of Rheumatology, 26, 1570-1576.

[8] Wolfe, F., Ross, K., Anderson, J., Russell, I.J. and Hebert, L. (1995) The Prevalence and Characteristics of Fibromyalgia in the General Population. Arthritis \& Rheumatism, 38, 19-28. http://dx.doi.org/10.1002/art.1780380104

[9] Jones, K.D., Horak, F.B., Winters-Stone, K., Irvine, J.M. and Bennett, R.M. (2009) Fibromyalgia Is Associated with Impaired Balance and Falls. Journal of Clinical Rheumatology, 15, 16-21. http://dx.doi.org/10.1097/RHU.0b013e318190f991

[10] Jones, K.D., King, L.A., Mist, S.D., Bennett, R.M. and Horak, F.B. (2011) Postural Control Deficits in People with Fibromyalgia: A Pilot Study. Arthritis Research \& Therapy, 13, R127. http://dx.doi.org/10.1186/ar3432

[11] Russek, L.N. and Fulk, G.D. (2009) Pilot Study Assessing Balance in Women with Fibromyalgia Syndrome. Physiotherapy Theory and Practice, 25, 555-565. http://dx.doi.org/10.3109/09593980802668050

[12] Bayazit, Y.A., Gursoy, S., Ozer, E., Karakurum, G. and Madenci, E. (2002) Neurotologic Manifestations of the Fibromyalgia Syndrome. Journal of the Neurological Sciences, 196, 77-80. http://dx.doi.org/10.1016/S0022-510X(02)00032-1

[13] Hargrove, J.B., Bennett, R.M., Simons, D.G., Smith, S.J., Nagpal, S. and Deering, D.E. (2010) Quantitative Electroencephalographic Abnormalities in Fibromyalgia Patients. Clinical EEG and Neuroscience, 41, 132-139. http://dx.doi.org/10.1177/155005941004100305

[14] Treede, R.D., Apkarian, A.V., Bromm, B., Greenspan, J.D. and Lenz, F.A. (2000) Cortical Representation of Pain: Functional Characterization of Nociceptive Areas Near the Lateral Sulcus. Pain, 87, 113-119. http://dx.doi.org/10.1016/S0304-3959(00)00350-X

[15] Nashner, L.M. and Peters, J.F. (1990) Dynamic Posturography in the Diagnosis and Management of Dizziness and Balance Disorders. Neurologic Clinics, 8, 331-349.

[16] Chien, C.W., Hu, M.H., Tang, P.F., Sheu, C.F. and Hsieh, C.L. (2007) A Comparison of Psychometric Properties of the Smart Balance Master System and the Postural Assessment Scale for Stroke in People Who Have Had Mild Stroke. Archives of Physical Medicine and Rehabilitation, 88, 374-380. http://dx.doi.org/10.1016/j.apmr.2006.11.019

[17] Dickin, D.C. and Rose, D.J. (2004) Sensory Organization Abilities during Upright Stance in Late-Onset Alzheimer'sType Dementia. Experimental Aging Research, 30, 373-390. http://dx.doi.org/10.1080/03610730490484416

[18] Ford-Smith, C.D., Wyman, J.F., Elswick Jr., R.K., Fernandez, T. and Newton, R.A. (1995) Test-Retest Reliability of the Sensory Organization test in Noninstitutionalized Older Adults. Archives of Physical Medicine and Rehabilitation, 76, 77-81. http://dx.doi.org/10.1016/S0003-9993(95)80047-6 
[19] Rose, D. (2010) Fallproof. A Comprehensive Balance and Mobility Training Program. 2nd Edition, Human Kinetics, Champaign.

[20] Shumway-Cook, A., Woollacott, M., Kerns, K.A. and Baldwin, M. (1997) The Effects of Two Types of Cognitive Tasks on Postural Stability in Older Adults with and without a History of Falls. The Journals of Gerontology Series A: Biological Sciences and Medical Sciences, 52, M232-M240. http://dx.doi.org/10.1093/gerona/52A.4.M232

[21] Teasdale, N., Bard, C., LaRue, J. and Fleury, M. (1993) On the Cognitive Penetrability of Posture Control. Experimental Aging Research, 19, 1-13. http://dx.doi.org/10.1080/03610739308253919

[22] Shumway-Cook, A. and Woollacott, M. (2000) Attentional Demands and Postural Control: The Effect of Sensory Context. The Journals of Gerontology Series A: Biological Sciences and Medical Sciences, 55, M10-M16. http://dx.doi.org/10.1093/gerona/55.1.M10

[23] Dick, B., Verrier, M., Harker, K. and Rashiq, S. (2008) Disruption of Cognitive Function in Fibromyalgia Syndrome. Pain, 139, 610-616. http://dx.doi.org/10.1016/j.pain.2008.06.017

[24] Cherry, B.J., Zettel-Watson, L., Shimizu, R., Roberson, I., Rutledge, D.N. and Jones, C.J. (2012) Cognitive Performance in Women Aged 50 Years and Older with and without Fibromyalgia. The Journals of Gerontology Series B: Psychological Sciences and Social Sciences, 69, 199-208.

[25] Dick, B., Eccleston, C. and Crombez, G. (2002) Attentional Functioning in Fibromyalgia, Rheumatoid Arthritis, and Musculoskeletal Pain Patients. Arthritis Care \& Research, 47, 639-644. http://dx.doi.org/10.1002/art.10800

[26] NeuroCom (1991) Equitest System Data Interpretation Manual. NeuroCom International Inc., Clackamas.

[27] Marsh, K. (2011) Reliability of the Sensory Organization Test ${ }^{\circledR}$ as a Measure of Sensory Reception in Older Adults with Fibromyalgia. (M.S. 1501510), California State University, Fullerton, United States -California. Dissertations \& Theses @ California State University, Fullerton Database. http://search.proquest.com/docview/893659676?accountid=9840

[28] Benton, A., Hamsher, K. and Sivan, A. (1994) Multilingual Aphasia Examination. AJA Associates, Iowa City.

[29] Chong, R.K.Y., Mills, B., Dailey, L., Lane, E., Smith, S. and Lee, K-H. (2010) Specific Interference between a Cognitive Task and Sensory Organization for Stance Balance Control in Healthy Young Adults: Visuospatial Effects. Neuropsychologia, 48, 2709-2718. http://dx.doi.org/10.1016/j.neuropsychologia.2010.05.018

[30] Busch, A., Webber, S., Brachaniec, M., Bidonde, J., Bello-Haas, V., Danyliw, A. and Schachter, C. (2011) Exercise Therapy for Fibromyalgia. Current Pain and Headache Reports, 15, 358-367. http://dx.doi.org/10.1007/s11916-011-0214-2 